\title{
Relations Between Summation Methods and Integral Transformations ${ }^{1}$
}

\author{
Werner Greub ${ }^{2}$ \\ (May 12, 1959)

\begin{abstract}
Relations between the Lototsky method of summation and those of Borel, Euler, and Knopp are obtained by associating an integral transformation with the series transformation.
\end{abstract}

\section{Introduction}

In a recent paper, R. P. Agnew [1] ${ }^{3}$ establishes relations between the Lototsky method of summation for divergent series and the classical summations of Borel, Euler, and Knopp.

It is the purpose of this paper to show that these relations can be obtained in a very natural way if the series transformation is associated with an integral transformation. The following scheme describes the connections between the series and the integral transformation:

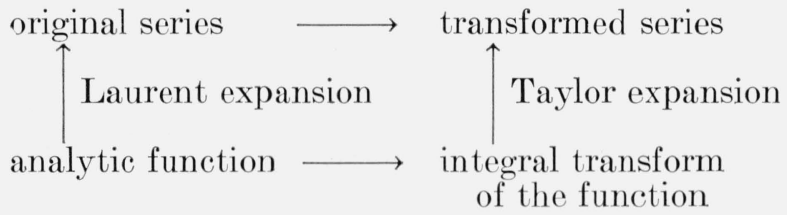

In section 2 it is shown that under certain conditions a regular series transformation can be assigned to every integral transformation, and conversely. As an example, the integral transformation corresponding to the Lototsky series transformation (L-transformation) is constructed. This integral transformation is used in section 3 for the discussion of the relation between the Lototsky and the Borel integral methods. The outcome of this discussion is that L-summability contains Borel integral-summability (BI-summability) but does not contain a modified Borel summability (BI*-summability as denoted in [1]). It is shown that the L-method can be extended to a summation method which is equivalent to the BI*-method.

In section 4 it is proved that a power series is L-summable in all interior points of the Borel polygon of the corresponding analytic function but not L-summable in all the exterior points. This proof is based on the relationship between the L-method and the BI-method.

In section 5 the Lototsky method is compared with the method of Euler-Knopp.

In the last section the inverse of the L-integral transform is constructed and used to obtain the inverse matrix of the Lototsky series transformation.

\footnotetext{
1 This work was carried out in part under a National Bureau of Standards

contract with The A merican University.
2 Present address: University of Zürich, Zürich, Switzerland.

${ }_{3}^{2}$ Present address: University of Zürich, Zürich, Switzerland.
}

\section{General Relations Between Series and Integral Transforms}

Let $a_{n v}(n, v=1,2, \ldots)$ be an infinite matrix whose elements are complex and subject to the condition

$$
\varlimsup_{v \rightarrow \infty} \sqrt[v]{\left|a_{n v}\right|}=0 \quad(n=1,2, \ldots) .
$$

We consider a not necessarily convergent infinite series

$$
\sum_{v=1}^{\infty} u_{v}
$$

with the property

$$
\varlimsup_{v \rightarrow \infty} \sqrt[v]{\left|u_{v}\right|}<\infty .
$$

This condition assures that the radius of convergence of the power series

$$
\sum_{v=1}^{\infty} \frac{u_{v}}{z_{v}}
$$

is positive.

Under conditions (1) and (3) all the series

$$
U_{n}=\sum_{v=1}^{\infty} a_{n v} u_{v}
$$

are convergent, as can readily be seen with the help of the root test. Therefore, we can say that under those conditions the matrix $\left(a_{n v}\right)$ defines the series transformation

$$
u_{n} \rightarrow U_{n}=\sum_{v=1}^{\infty} a_{n v} u_{v} .
$$

We will show now that one can associate with this series transformation a certain integral transformation between the analytic functions

$$
f(z)=\sum_{v=1}^{\infty} \frac{u_{v}}{z^{v}}
$$

and

$$
\phi(\alpha)=\sum_{n=1}^{\infty} U_{n} \alpha^{n} .
$$


'I'o obtain the kernel of this transformation we define the functions $P_{n}(z)$ by

$$
P_{n}(z)=\sum_{n=0}^{\infty} a_{n v+1} z^{v} \quad(n=1,2, \ldots) .
$$

Because of (1) these series are convergent for every $z$ and thus $P_{n}(z)$ are entire functions of $z$.

It follows from (3) that the function $f(z)$ is regular outside of a certain circle, $|z|=\rho$. Now we integrate the product, $P_{n}(z) f(z)$ along a circle $|z|=R \quad(R>\rho)$, and obtain

$$
\begin{aligned}
& \phi P_{n}(z) f(z) d z=\oint \sum_{v, \mu} a_{n v+1} u_{v} z^{v_{\mu}} d z \\
&=2 \pi i \sum_{\mu=1}^{\infty} a_{n \mu} u_{\mu}=2 \pi i U_{n} .
\end{aligned}
$$

Under the hypothesis that the function

$$
K(z, \alpha)=\sum_{n=1}^{\infty} P_{n}(z) \alpha^{n}
$$

is regular in the domain $|z|<\infty,|\alpha|<1$, we derive from $(6)$

$$
\oint K(z, \alpha) f(z) d z=2 \pi i \sum_{n=1}^{\infty} U_{n} \alpha^{n} .
$$

Hence, defining the function $\phi(\alpha)$ for $|\alpha|<1$ by

$$
\phi(\alpha)=\sum_{n=1}^{\infty} u_{n} \alpha^{n}
$$

we obtain the integral transformation ${ }^{4}$

$$
\phi(\alpha)=\frac{1}{2 \pi i} \oint K(z, \alpha) f(z) d z
$$

which maps every function $f(z)$ which is regular outside of a certain circle into a function $\phi(\alpha)$ which is regular within the unit circle.

Conversely, let (7) be an integral transformation whose kernel $K(z, \alpha)$ is regular in the domain $|z|<\infty, \quad|\alpha|<1$ and fulfills the condition ${ }^{5} K(z, 0)=0$. Substituting for $f(z)$ a function ${ }^{6}$ regular for $|z|>1$,

$$
f(z)=\sum_{v=1}^{\infty} \frac{u_{v}}{z^{v}}
$$

we obtain for $\phi(\alpha)$ a function which can be expanded into a power series within the unit circle,

$$
\phi(\alpha)=\sum_{n=1}^{\infty} U_{n} \alpha^{n}
$$

\footnotetext{
4 The path of integration in the $z$-plane will always be a circle $|z|=R \quad(R>1)$. 5 This is not an essential restriction because it can be achieved by replacing $K(z, \alpha)$ by $K(z, \alpha)-K(z, 0)$. Then the function $\phi(\alpha)$ is only changed by a con-

${ }^{6}$ It is assumed that there is no constant term in the expansion; in other words, that $f(\infty)=0$.
}

From (7) we obtain between the coefficients $u_{v}$ and $U_{n}$ the relations

$$
\begin{aligned}
U_{n}=\frac{1}{2 \pi i} \oint \frac{\phi(\alpha)}{\alpha^{n+1}} & d \alpha=\left(\frac{1}{2 \pi i}\right)^{2} \oint \oint \frac{K(z, \alpha)}{\alpha^{n+1} z^{v}} f(z) d z d \alpha \\
& =\left(\frac{1}{2 \pi i}\right)^{2} \oint \oint \sum_{v} \frac{K(z, \alpha)}{\alpha^{n+1}} \frac{u_{v}}{z^{v}} d z d \alpha .
\end{aligned}
$$

Hence the integral transformation (7) defines a series transformation whose matrix is given by

$$
a_{n v}=\left(\frac{1}{2 \pi i}\right)^{2} \oint \oint \frac{K(z, \alpha)}{\alpha^{n+1} z^{v}} d z d \alpha .
$$

It can be shown that under certain hypotheses about the kernel the series transformation (8) is regular in the following sense: If the series $\sum_{v} u_{v}$ is convergent, the series $\sum_{n} U_{n}$ is Abelian summable (A-summable). This means that the limit

$$
\lim _{\alpha \rightarrow 1-0}\left(\sum_{n=1}^{\infty} U_{n} \alpha^{n}\right) \quad(\alpha \text { real })
$$

exists and the relation

$$
\lim _{\alpha \rightarrow 1-0}\left(\sum_{n=1}^{\infty} U_{n} \alpha^{n}\right)=\sum_{v=1}^{\infty} u_{v}
$$

holds. The hypotheses about the kernel are:

(i) $K(z, \alpha)$ is a regular function of $z$ and $\alpha$ in the domain $|z|<\infty,|\alpha|<1$ and therefore can be expanded in the form

$$
K(z, \alpha)=\sum_{n=0}^{\infty} a_{n+1}(\alpha) z^{n}
$$

(ii) For every fixed $n$ we have

$$
\lim _{\alpha \rightarrow 1-0} a_{n}(\alpha)=1 \quad(\alpha \text { real })
$$

(iii) There exists a constant $M$ not depending on $\alpha$ such that

$$
\sum_{n=1}^{\infty}\left|a_{n+1}(\alpha)-a_{n}(\alpha)\right| \leqq M
$$

To prove the above statement we consider the transformation

$$
\phi(\alpha)=\frac{1}{2 \pi i} \oint K(z, \alpha) f(z) d(z)
$$

and substitute for $K(z, \alpha)$ and $f(z)$ the expansions (9) and (5), respectively. So we obtain the seriesto-function transformation

$$
\phi(\alpha)=\sum_{v=1}^{\infty} u_{v} a_{v}(\alpha)
$$


Using a well-known regularity theorem we conclude from conditions (ii) and (iii) that this transformation is regular; this means, the $\operatorname{limit} \lim \phi(\alpha)$ exists provided that the series $\sum_{v} u_{v}$ is convergent and the relation

$$
\lim _{\alpha \rightarrow 1-0} \phi(\alpha)=\sum_{v=1}^{\infty} u_{v}
$$

holds.

The statement which has just been proved can be formulated as the following:

Theorem 1. Let $K(z, \alpha)$ be a function with the properties (i), (ii), and (iii), and let the matrix $a_{n v}$ be defined by

$$
a_{n v}=\left(\frac{1}{2 \pi i}\right)^{2} \oint \oint \frac{K(z, \alpha)}{\alpha^{n+1} z^{v}} d z d \alpha .
$$

Then the series transformation induced by this matrix carries every convergent series $\sum_{v} u_{v}$ into an A-summable series $\sum_{n} U_{n}$ with the same sum.

It is worth noting that the series $\sum_{n} U_{n}$ need not be convergent itself. However, if the matrix $a_{n v}$ fulfills additional inequalities of the form

$$
\sum_{v=1}^{\infty}\left|a_{n v}\right| \leqq \frac{M}{n} \quad(n=1,2, \ldots)
$$

where $M$ is a fixed number, it follows that

$$
\left|U_{n}\right| \leqq \frac{M}{n}
$$

In this case we can apply the Tauberian theorem and conclude that the series $\sum_{n} U_{n}$ is convergent and

$$
\sum_{n} U_{n}=\lim _{\alpha \rightarrow 1-0} \sum_{n}\left(U_{n} \alpha^{n}\right) .
$$

Thus, under the additional hypothesis (12), we get

$$
\sum_{n} U_{n}=\sum_{0} u_{0}
$$

for every convergent series $\sum_{v} u_{v}$, which means that the transformation (4) is regular.

As an example, let us consider the series transformation

$$
U_{1}=u_{1}, \quad U_{n}=\frac{1}{n}, \sum_{v=1}^{n} p_{n-1, v-1} u_{v}
$$

where the $p_{n v}$ are the coefficients of the polynomial

$$
p_{n}(z)=z(z+1) \ldots(z+n-1) \quad(n=1,2, \ldots) .
$$

This transformation was introduced by Lototsky [3] and will be called the L-transformation. If the series $\sum_{n} U_{n}$ is convergent respectively $\mathrm{A}$-summable the original series $\sum_{v} u_{v}$ will be called L-summable respectively AL-summable, and we write

$$
\sum_{n} U_{n}=L\left(\sum_{v} u_{v}\right)
$$

and

$$
\lim _{\alpha \rightarrow 1-0}\left(\sum_{n} U_{n} \alpha^{n}\right)=A L\left(\sum_{v} u_{v}\right) .
$$

For the integral kernel, $K(z, \alpha)=\sum_{n=1}^{\infty} P_{n}(z) \alpha^{n}$, of the L-transformation, the functions $Y_{n}(z)$ have the following form:

$P_{1}(z)=z$

$P_{n}(z)=\frac{1}{n !} p_{n-1}(z)=\frac{(-1)^{n}}{z-1}\left(\begin{array}{c}1-z \\ n\end{array}\right) \quad(n=2,3, \ldots)$.

This leads to

$$
K(z, \alpha)=\frac{1}{z-1} \sum_{n=1}^{\infty}(-1)^{n}\left(\begin{array}{c}
1-z \\
n
\end{array}\right) \alpha^{n}=\frac{(1-\alpha)^{1-z}-1}{z-1} .
$$

Hence, defining the analytic function $\phi(\alpha)$ $(|\alpha|<1)$ by

$$
\phi(\alpha)=\frac{1}{2 \pi i} \oint \frac{(1-\alpha)^{1-z}-1}{z-1} f(z) d z,
$$

the AL-summability of the series $\sum_{v} u_{0}$ means that $\phi(\alpha)$ approaches a limit if $\alpha$ tends to 1 from the left along the real axis and we have the relation

$$
A L\left(\sum_{v} u_{v}\right)=\lim _{\alpha \rightarrow 1-0} \phi(\alpha) .
$$

In order to show that a convergent series is also AL-summable to the same value we check that the kernel $K(z, \alpha)$ satisfies the hypothesis of theorem 1 . Condition (i) is immediately clear and (ii) follows from the fact that $a_{n}(\alpha)$ can be written as

$$
a_{n}(\alpha)=\frac{1}{2 \pi i} \oint \frac{K(z, \alpha)}{z^{n}} d z=1-e^{-\gamma} \sum_{v=1}^{n} \frac{\gamma^{v}}{v !}
$$

where

$$
\gamma=-\log (1-\alpha)
$$

To check (iii) we observe that

$$
a_{n+1}(\alpha)-a_{n}(\alpha)=e^{-\gamma} \frac{\gamma^{n+1}}{(n+1) !},
$$


and therefore

$$
\begin{aligned}
\sum_{n=1}^{\infty}\left|a_{n+1}(\alpha)-a_{n}(\alpha)\right|=e^{-\gamma} \sum_{n=1}^{\infty} & \frac{\gamma^{n+1}}{(n+1) !} \\
& <e^{-\gamma} e^{\gamma}=1 \quad(0 \leqq \alpha<1) .
\end{aligned}
$$

Now it follows from theorem 1 that the transformation (13) maps a convergent series into an A-summable series with the same sum.

In this special case it even can be shown that the series $\sum_{n} U_{n}$ is convergent itself. From (13) we get

$$
\sum_{v=1}^{\infty} a_{n v}=\frac{1}{n !} \sum_{v=1}^{n} p_{n-1} p_{v-1}=\frac{1}{n !} p_{n-1}(1)=\frac{1}{n},
$$

and thus condition (12) is fulfilled which assures the convergence of the series $\sum_{n} U_{n}$. This means that the L-method is regular.

\section{Connections Between the Lototsky and the Borel Summabilities}

As mentioned before, there is a close relationship between the Lototsky and the Borel-integral methods of summation. Let $\sum u_{v}$ be a (not necessarily convergent) infinite series such that

$$
\varlimsup_{n \rightarrow \infty} \sqrt[n]{\frac{\left|u_{n}\right|}{n !}}=0 .
$$

'Then the sum

$$
F(t)=\sum_{t=0}^{\infty} \frac{u_{v+1}}{v !} t^{v}
$$

defines an entire function of $t$. The series $\sum_{v} u_{v}$ is called summable with respect to the Borel integral method (BI-summable) if the improper integral

$$
\int_{0}^{\infty} e^{-t} F(t) d t
$$

exists. In this case we write

$$
B I\left(\sum_{v} u_{v}\right)=\int_{0}^{\infty} e^{-t} F(t) d t .
$$

We are going to show that the function

$$
B(t)=\int_{0}^{t} e^{-\tau} F(\tau) d \tau
$$

can be represented as a certain integral transform of the function

$$
f(z)=\sum_{v=1}^{\infty} \frac{u_{v}}{z^{v}}
$$

Assume (as we did in sec. 2) that the radius of convergence of the series (21) is positive; in other words, that

$$
\varlimsup_{n \rightarrow \infty} \sqrt[n]{\left|u_{n}\right|}=\frac{1}{r}<\infty
$$

Since $\sqrt[n]{n !} \rightarrow \infty$ it follows from (22) that condition (18) is automatically fulfilled.

The coefficients $u_{n}$ are represented by the integrals

$$
u_{n+1}=\frac{1}{2 \pi i} \oint f(z) z^{n} d z
$$

where the integral is taken around a certain circle $|z|=R \quad(R>r)$. From (23) it follows that

$$
\sum_{n=0}^{\infty} \frac{u_{n+1}}{n !} t^{n}=\frac{1}{2 \pi i} \oint f(z) \sum_{n=0}^{\infty} \frac{z^{n} t^{n}}{n !} d z=\frac{1}{2 \pi i} \oint f(z) e^{z t} d z
$$

and therefore, using (19) and (20),

$$
B(t)=\frac{1}{2 \pi i} \int_{0}^{t} \oint e^{\tau(z-1)} f(z) d z d \tau .
$$

Since the integrand is regular we can interchange the two integrations and integrating with respect to $\tau$ we obtain

$$
B(t)=\frac{1}{2 \pi i} \oint \frac{e^{t(z-1)}-1}{z-1} f(z) d z .
$$

Thus the BI-sum of the series $\sum_{v} u_{v}$ can be represented as the limit

$$
B I\left(\sum_{v} u_{v}\right)=\lim _{t \rightarrow \infty} \frac{1}{2 \pi i} \oint \frac{e^{t(z-1)}-1}{z-1} f(z) d z .
$$

Comparing transformations (16) and (25), we obtain the following relation between functions $\phi(\alpha)$ and $B(t)$ :

$$
\phi(\alpha)=B(-\log (1-\alpha)) \quad(|\alpha|<1),
$$

which can also be written as

$$
B(t)=\phi\left(1-e^{-t}\right) .
$$

From this relation it follows immediately that the existence of one of the limits

$$
\lim _{\alpha \rightarrow 1-0} \phi(\alpha) \quad \text { or } \quad \lim _{t \rightarrow \infty} B(t)
$$

implies the existence of the other, and these limits coincide. This means in terms of series: Under the hypothesis (22) AL- and the BI-summability are equivalent.

Here the question arises whether this equivalence still holds without condition (22). We are going to show that the implication $\mathrm{BI} \rightarrow \mathrm{AL}$ is true in general but not conversely. 
We first prove

Lemma 1: Let $u_{v}$ be an infinite series such that the function

$$
F(t)=\sum_{v=0}^{\infty} \frac{u_{v+1}}{v} t^{v}
$$

is regular in a certain neighborhood of $t=0$ and define the functions $B(t)$ and $\phi(\alpha)$ by

$$
B(t)=\int_{0}^{t} F(\tau) e^{-\tau} d \tau
$$

and

$$
\phi(\alpha)=B(-\log (1-\alpha)) .
$$

Then the coefficients $U_{n}$ of the expansion

$$
\phi(\alpha)=\sum_{v} U_{n} \alpha^{n}
$$

are the L-transforms of $u_{v}$.

Proof: The coefficients $U_{n}$ are defined by

$$
B(-\log (1-\alpha))=\sum_{n} U_{n} \alpha^{n} .
$$

Differentiating this relation we get

$$
B^{\prime}(-\log (1-\alpha)) \frac{1}{1-\alpha}=\sum_{n} n U_{n} \alpha^{n-1},
$$

and hence, observing that

$$
B^{\prime}(t)=F(t) e^{-t}
$$

and therefore

$$
B^{\prime}(-\log (1-\alpha))=(1-\alpha) F(-\log (1-\alpha)),
$$

we conclude that

$$
\sum_{n} n U_{n} \alpha^{n-1}=\sum_{v=0}^{\infty} \frac{(-1)^{v}}{v !}[\log (1-\alpha)]^{v} u_{v+1}
$$

Using the residue theorem we conclude that

$$
U_{n}=\frac{1}{n} \sum_{v=0}^{\infty} \frac{(-1)^{v}}{v !}\left(\oint \frac{[\log (1-\alpha)]^{v}}{\alpha^{n+1}} d \alpha\right) u_{v+1},
$$

which can be written as

$$
U_{n}=\frac{1}{n !} \sum_{v=0}^{\infty} a_{n-1} v u_{v+1}=\frac{1}{n !} \sum_{v=1}^{\infty} a_{n-1}{ }_{v-1} u_{v}
$$

where the coefficients $a_{n v}$ are defined by

$$
a_{n v}=(-1)^{v} \frac{n !}{v !} \oint \frac{[\log (1-\alpha)]^{v}}{\alpha^{n+1}} d \alpha .
$$

It has to be shown that the numbers $a_{n v}$ coincide with the numbers $p_{n v}$ defined by (14). First of all we have $a_{n v}=0$ for $v>n$ since the functions

$$
\frac{[\log (1-\alpha)]^{v}}{\alpha^{n+1}}
$$

are regular at $\alpha=0$ provided that $v \geqq n+1$. We now define the polynomials $a_{n}(z)$ by

and show that

$$
a_{n}(z)=\sum_{v=1}^{n} a_{n v} z^{v}
$$

$$
a_{n}(-k)=0 \quad(k=0,1, \ldots, n-1) .
$$

In fact we have for every integer

$$
\begin{aligned}
\frac{1}{n !} a_{n}(-k) & =\sum_{v=1}^{n} \frac{k^{v}}{v !} \oint \frac{[\log (1-\alpha)]^{v}}{\alpha^{n+1}} d \alpha \\
& =\sum_{v=1}^{\infty} \frac{k^{v}}{v !} \oint \frac{[\log (1-\alpha)]^{v}}{\alpha^{n+1}} d \alpha=\oint \frac{(1-\alpha)^{k}-1}{\alpha^{n+1}} d \alpha .
\end{aligned}
$$

If now $k \leqq n-1$ there is no residue at $\alpha=0$ and we get

$$
a_{n}(-k)=0 \quad(k=0,1, \ldots, n-1) .
$$

From here it follows, since the polynomial $a_{n}(z)$ has the degree $n$,

$$
a_{n}(z)=\lambda z(z+1) \ldots(z+n-1)
$$

where $\lambda$ is a constant. Substituting $z=1$ in (33) we get

$$
a_{n}(1)=\lambda n !
$$

and on the other hand we have

$$
\begin{aligned}
a_{n}(1)=\sum_{v=1}^{n} a_{n v} & =\sum_{v=1}^{\infty} a_{n v}=n ! \sum_{v=1}^{\infty} \frac{(-1)^{v}}{v !} \oint \frac{[\log (1-\alpha)]^{v}}{\alpha^{n+1}} d \alpha \\
& =n ! \oint\left(\frac{1}{1-\alpha}-1\right) \frac{d \alpha}{\alpha^{n+1}}=n !
\end{aligned}
$$

From the last two relations it follows that $\lambda=1$ and we obtain

$$
a_{n}(z)=z(z+1) \ldots(z+n-1)=p_{n}(z)
$$

and comparing the coefficients

$$
a_{n v}=p_{n v},
$$

which proves lemma 1. 
Theorem $2 .^{7}$ A BI-summable series is also $A L$ summable and the two sums coincide:

$$
B I\left(\sum_{v} u_{v}\right)=A L\left(\sum_{v} u_{v}\right)
$$

Proof: By hypothesis the series

$$
F(t)=\sum_{v=0}^{\infty} \frac{u_{v+1}}{v !} t^{v}
$$

defines an entire function. Hence the function

$$
B(t)=\int_{0}^{t} F(\tau) e^{-\tau} d t
$$

is entire too, and therefore the function

$$
\phi(\alpha)=B(-\log (1-\alpha))
$$

is regular for $|\alpha|<1$. According to lemma 1 we have the expansion

$$
\phi(\alpha)=\sum_{n=1}^{\infty} U_{n} \alpha^{n}
$$

where the $U_{n}$ are the L-transforms of the $u_{v}$. Hence the series

$$
\sum_{n=1}^{\infty} U_{n} \alpha^{n}
$$

is convergent for $|\alpha|<1$. Furthermore, we obtain from (34) and (35)

$$
\sum_{n=1}^{\infty} U_{n} \alpha^{n}=B(-\log (1-\alpha))
$$

and hence, if $\alpha$ tends to 1 on the real axis,

$$
\lim _{\alpha \rightarrow 1-0}\left(\sum_{n=1}^{\infty} U_{n} \alpha^{n}\right)=\lim _{t \rightarrow+\infty} B(t) .
$$

This means that the series $\sum_{v} u_{v}$ is AL-summable and that the relation

$$
A L\left(\sum_{v} u_{v}\right)=B I\left(\sum_{v} u_{v}\right)
$$

holds.

Conversely, one cannot conclude that an ALsummable (even an L-summable) series is also BI-summable, as the following example shows:

Let the function $\varphi(t)$ be defined by

$$
\varphi(t)=\frac{1}{2-e^{-t}}
$$

${ }^{7}$ Different proofs of theorems 2 to 4 are given in a mimeographed report by R. P. Agnew (U.S.A.F. Contract $18(600-685))$. and the sequence $u_{n}$ by

$$
u_{n}=\varphi^{(n-1)}(0) \quad(n=1,2, \ldots) .
$$

Then we have

$$
\sum_{v=0}^{\infty} \frac{u_{v+1}}{v !} t^{v}=\frac{1}{2-e^{-t}}
$$

and therefore this series is convergent only for $|t|<\log 2$. Hence the BI-method cannot be applied.

To obtain the L-transform of sequence (36) we apply lemma 1. The function $B(t)$ in our case is given by

$$
B(t)=\int_{0}^{t} \frac{e^{-\tau} d \tau}{2-e^{-\tau}}=\log \left(2-e^{-t}\right)
$$

and from here we get

$$
\phi(\alpha)=B(-\log (1-\alpha))=\log (1+\alpha),
$$

and thus

$$
U_{n}=(-1)^{n-1} \frac{1}{n} .
$$

The series $\sum_{n} U_{n}$ is convergent, and hence series (36) is L-summable to the value $\log 2$.

As the above example shows, the AL-summability of a series $\sum_{v} u_{v}$ does not imply that the series

$$
\sum_{v} \frac{u_{v+1}}{v !} t^{v}
$$

has an infinite radius of convergence. It can be shown however that this radius can not be less than $\log 2$. To this purpose we prove

Lemma 2 : Let $\sum_{v} u_{v}$ be an infinite series and $\sum_{n} U_{n}$ its L-transform. Assume that the series

$$
\phi(\alpha)=\sum_{v} U_{n} \alpha^{n}
$$

is convergent in a certain circle $|\alpha|<\rho,(\rho>0)$. Then the series

$$
F(t)=\sum_{v} \frac{u_{v+1}}{v !} t^{v}
$$

is convergent in a certain circle $|t|\left\langle\rho^{\prime},\left(\rho^{\prime}>0\right)\right.$ and the relation

$$
\int_{0}^{t}\left(\sum_{v} \frac{u_{v+1}}{v !} \tau^{v}\right) e^{-\tau} d \tau=\phi\left(1-e^{-t}\right)
$$

holds.

Proof: By hypothesis the function

$$
B(t)=\phi\left(1-e^{-t}\right)
$$

is regular in a certain circle $|t|\left\langle\rho^{\prime}\right.$ and therefore the same is true for the function

$$
F(t)=B^{\prime}(t) e^{t},
$$


where $B^{\prime}$ indicates the derivative of $B$.

From (38) and (39) it follows that

$$
F(t)=\phi^{\prime}\left(1-e^{t}\right) \cdot
$$

Expanding the function $F(t)$ into a power series,

$$
\left.F(t)=\sum_{v}\right\lrcorner c_{v} t^{v},
$$

we obtain from (40)

$$
\phi^{\prime}\left(1-e^{-t}\right)=\sum_{v} c_{v} t^{v},
$$

and if we introduce

$$
\alpha=1-e^{-t}
$$

as a new variable,

$$
\phi^{\prime}(\alpha)=\sum_{v}(-1)^{v} c_{v}[\log (1-\alpha)]^{v} .
$$

On the other hand we have

$$
\phi^{\prime}(\alpha)=\sum_{n} n U_{n} \alpha^{n-1} .
$$

From (41) and (42) it follows that

$$
\sum_{n} n U_{n} \alpha^{n-1}=\sum_{v}(-1)^{v} c_{v}[\log (1-\alpha)]^{v},
$$

and from here we obtain

$$
\begin{aligned}
U_{n}=\frac{1}{2 \pi i} i \frac{1}{n} \sum_{v}(-1)^{v} c_{v} \oint \frac{[\log (1-\alpha)]^{v}}{\alpha^{n}} & d \alpha \\
& =\frac{1}{n !} \sum_{v} \rho_{n-1} v ! c_{v} .
\end{aligned}
$$

This equation states that the $U_{n}$ are the Ltransforms of the sequence $v ! c_{v}$. On the other hand, they are by definition the L-transforms of $u_{v}$, and hence we conclude

$$
c_{v}=\frac{1}{v !} u_{v+1} .
$$

Therefore the expansion of $F(t)$ becomes

$$
F(t)=\sum_{v=0}^{\infty} \frac{u_{v+1}}{v !} t^{\imath}
$$

and this series must be convergent within the circle $|t|<\rho^{\prime}$.

Relation (37) now follows from (43), (39), and (38).

From lemma 2 we can derive the following theorem, which is proved by Agnew [2] in a different way.

Theorem. For the terms of an AL-summable series $\sum_{v} u_{v}$ the inequality

$$
\varlimsup_{n \rightarrow \infty} \sqrt[n]{\frac{\left|u_{n}\right|}{n !}} \leqq \frac{1}{\log 2}
$$

holds.

Proof: Since the series $\sum_{v} u_{0}$ is AL-summable the function

$$
\phi(\alpha)=\sum_{n} U_{n} \alpha^{n}
$$

is regular for $|\alpha|<1$. Hence the function $B(t)$ defined by

$$
B(t)=\phi\left(1-e^{-t}\right)
$$

must be regular in the domain $D$, which is determined by the inequality

$$
D:\left|1-e^{-t}\right|<1
$$

and the same holds for the function

$$
F(t)=B^{\prime}(t) e^{-t} .
$$

Writing $t=x+i y$, we get

$$
\left|1-e^{-t}\right|^{2}=1+2 e^{-x}\left(\frac{1}{2} e^{-x}-\cos y\right)
$$

and hence inequality (45) is equivalent to

$$
\frac{1}{2} e^{-x}<\cos y .
$$

This means that the point $t=x+i y$ lies in the "interior" of the curve

$$
x=-\log (2 \cos y) .
$$

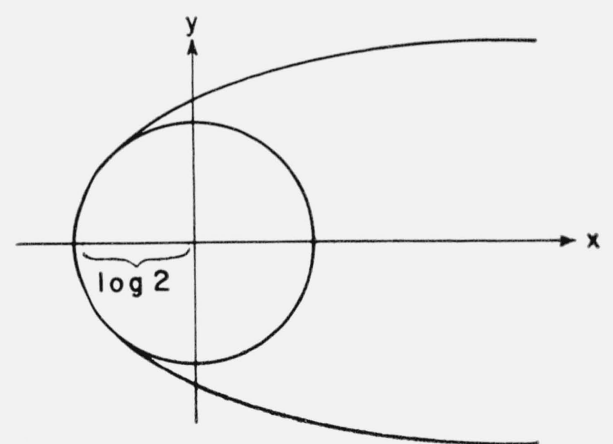

FIGURE 1. Relations between summation methods and integral transformations.

Thus the function $B(t)$ can be expanded into a power series within the largest circle $|t|=\rho$ with the center $t=0$ that is contained in the domain $D$. This is the circle

$$
|t|=\log 2 .
$$

because this circle touches curve (46) in the point $t=-\log 2$, it is sufficient to show that the radius of curvature of the curve (46) is not less than log 2 . The curvature of this curve is given by

$$
\frac{1}{\rho}=\frac{1}{\cos y}
$$


and hence we have

$$
\rho>1>\log 2 .
$$

Thus the expansion of $F(t)$ into a power series must be convergent for

$$
|t|<\log 2 .
$$

On the other hand, using lemma 2 this expansion is given by

$$
F(t)=\sum_{v} \frac{u_{v+1}}{v !} t^{v}
$$

From here it follows that

$$
\varlimsup_{n \rightarrow \infty} \sqrt[n]{\frac{\left|u_{n}\right|}{n !}} \leqq \frac{1}{\log 2}
$$

which proves theorem 3 .

It follows from series (36) that the bound 1/log 2 can not be improved.

The BI*-method. The application of the BImethod provides that the series

$$
\sum_{v} \frac{u_{v}+1}{v !} t^{v}
$$

converges in the whole $t$-plane. Frequently the case occurs that the radius of convergence of this series is positive and finite such that this series defines a regular function $F(t)$ within a certain circle $|t|<\rho$. If this function can be analytically continued along the positive $t$-axis one can form the improper integral

$$
\int_{0}^{\infty} F(t) e^{-t} d t
$$

In case this integral is convergent, the series is said to beBI*-summable [2, sec. 11$]$ and its value is called the $\mathrm{BI}^{*}$-sum of $\sum_{v} u_{v}$,

$$
B I^{*}\left(\sum_{v} u_{v}\right)=\int_{0}^{\infty} F(t) e^{-t} d t
$$

Obviously a BI-summable series is also BI*-summable to the same value.

It can be shown that AL-summability implies BI*-summability, but we do not prove this here since $\mathrm{BI}{ }^{*}$-summability will turn out to be equivalent to a generalization of AL-summability and the above statement will follow immediately from there.

To demonstrate the BI*-method by an example, let $u_{n}$ be defined by

$$
u_{n+1}=(-1)^{n} \frac{n !}{a^{n}} \quad(n=0,1,2, \ldots)
$$

where $a \quad(a \neq 0)$ is an arbitrary complex number. In this case we obtain

$$
\sum_{v} \frac{u_{v+1}}{v !} t^{v}=\frac{1}{1+t / a}=\frac{a}{a+t},
$$

and thus,

$$
B I^{*}\left(\sum_{v} u_{v}\right)=\int_{0}^{\infty} \frac{a e^{-t}}{a+t} d t .
$$

This integral converges for every number $a$ which is not real and negative and therefore series (47) is BI*-summable for all those values of $a$.

The next example shows that a BI*-summable series is not necessarily AL-summable (and hence not L-summable). Let the function $\phi(t)$ be defined by

$$
\phi(t)=\frac{1}{\left(a-e^{-t}\right)^{2}},
$$

where $a$ is a real number such that $a>1$, and let the sequence $u_{n}$ be defined by

$$
u_{n}=\phi^{(n-1)}(0) \quad(n=1,2, \ldots) .
$$

Then the series

$$
\sum_{v} \frac{u_{v}}{(v+1) !} t^{v}
$$

is convergent in the circle

$$
|t|<\log a
$$

and represents the function $\phi(t)$. This function is defined along the whole positive $t$-axis and the in tegral

$$
\int_{0}^{\infty} \frac{e^{-t} d t}{\left(a-e^{-t}\right)^{2}}=\frac{1}{a-1}-\frac{1}{a}
$$

is convergent. Thus the series (49) is BI*-summable.

In order to obtain the L-transform of (49) according to lemma 2 we have to form the function

$$
B(t)=\int_{0}^{t} \phi(\tau) e^{-\tau} d \tau=\frac{1}{a-1}-\frac{1}{a-e^{-t}}
$$

and then substitute

$$
t=-\log (1-\alpha) .
$$

This gives

$$
\phi(\alpha)=\frac{1}{a-1}-\frac{1}{a-1+\alpha} .
$$

The function $\phi(\alpha)$ so obtained has a pole at $\alpha=1-a$ and hence the series $\sum_{n} U_{n} \alpha^{n}$ is only convergent in
the circle

$$
|\alpha|<a-1,
$$


which is smaller than the unit circle if $a<2$. Therefore, the AL-method can not be applied to series (49).

The AL*-summability. As the last example shows the $\mathrm{BI}^{*}$-method is more powerful than the ALmethod. However the AL-method can be extended to a method $\mathrm{AL}^{*}$ in a similar way as $\mathrm{BI}$ has been extended to $\mathrm{BI} *$.

Let $\sum_{v} u_{v}$ be an infinite series such that the power series

$$
\phi(\alpha)=\sum_{n} U_{n} \alpha^{n}
$$

is convergent within a circle $|\alpha|<\rho$ and assume that the function $\phi(\alpha)$ can be analytically continued along the interval $0 \leqq u<1 \quad(\alpha=u+i v)$. If the limit

$$
\lim _{\alpha \rightarrow 1-0} \phi(\alpha) \quad(\alpha \text { real })
$$

exists we shall call the series $\sum_{v} u_{v} \mathrm{AL}^{*}$-summable and write

$$
\mathrm{AL}^{*}\left(\sum_{v} u_{v}\right)=\lim _{\alpha \rightarrow 1-0} \phi(\alpha)
$$

AL*-summability obviously implies AL-summability.

Theorem 4. The $A L^{*}$-summability is equivalent to the $B I^{*}$-summability.

Proof: Assume first the series $\left\{\sum_{n} u_{v}\right.$ is $\mathrm{BI}^{*}$-summable. Then the function

$$
F(t)=\sum_{v} \frac{u_{v+1}}{v !} t^{v}
$$

is regular within a circle $|t|<\rho$. Let now the functions $B(t)$ and $\phi(\alpha)$ be given by

$$
B(t)=\int_{0}^{t} F(\tau) e^{-\tau} d \tau
$$

and

$$
\phi(\alpha)=B(-\log (1-\alpha))
$$

Then $\phi(\alpha)$ is regular within a circle $|\alpha|<\rho^{\prime}$ and therefore it can be expanded into a power series

$$
\phi(\alpha)=\sum_{n} U_{n} \alpha^{n} .
$$

According to lemma 1 the coefficients $U_{n}$ are the L-transforms of the $u_{v}$. By hypothesis, the function $F(t)$ can be analytically continued along the positive $t$-axis and therefore the same is true for the function $B(t)$. Hence eq (50) defines an analytic continuation of the function $\phi(\alpha)$ along the real unit interval and we have

$$
\lim _{\alpha \rightarrow 1-0} \phi(\alpha)=\lim _{t \rightarrow \infty} B(t)
$$

This means that the series $\sum_{v} u_{v}$ is $A L^{*}$-summable and the relation

holds.

$$
\mathrm{AL} *\left(\sum_{v} u_{v}\right)=\mathrm{BI} *\left(\sum_{v} u_{v}\right)
$$

On the other hand, if the series $\sum_{v} u_{v}$ is $\mathrm{AL}^{*}$-summable, the function

$$
\phi(\alpha)=\sum_{n} U_{n} \alpha^{n}
$$

is regular within a circle $|\alpha|<\rho$. Hence the series

$$
F(t)=\sum_{v} \frac{u_{v+1}}{v !} t^{v}
$$

according to lemma 2 is convergent within a circle $|t|<\rho^{\prime}$, and the relation

$$
F(t)=\phi^{\prime}\left(1-e^{-t}\right) e^{t}
$$

holds. Since the function $\phi(\alpha)$ by hypothesis can be analytically continued along the real unit interval the same holds for $F(t)$ along the positive $t$-axis and relation (51) holds for every positive $t$. From there we obtain

$$
\int_{0}^{\infty} F(t) e^{-t} d t=\lim _{\alpha \rightarrow 1-0} \phi(\alpha)
$$

which shows that the series $\sum_{v} u_{v}$ is $\mathrm{BI}^{*}$-summable.

Altogether we have the following relations between the Lototsky and the Borel methods, where all the simple arrows are not invertable:

$$
\begin{array}{cc}
L \rightarrow \underset{\uparrow}{\mathrm{AL}} \rightarrow & \underset{\mathrm{AL}}{*} \\
\mathrm{BI} & \mathrm{BI}^{*}
\end{array}
$$

Series (36) shows that L does not imply BI, but it is not known to the author whether the implication $\mathrm{BI} \rightarrow \mathrm{L}$ holds

\section{Application to Analytic Continuation}

Let $g(z)$ be an analytic function regular in a domain $D$ which contains the origin and let

$$
g(z)=\sum_{v=1}^{\infty} u_{v} z^{v-1}
$$

be the expansion of $g(z)$ around the point $z=0$. It is a known result that the series (52) is BI-summable to the value $g(z)$ at every interior point of the Borel polygon $\pi$ of $g(z)$ and not BI-summable at every exterior point. Making use of the relationship between the BI- and the L-summability the same can be shown for the L-summability of series (52).

In order to do so we first observe that

$$
\varlimsup_{n \rightarrow \infty} \sqrt[n]{\left|u_{n} z_{0}^{n-1}\right|}=\left|z_{0}\right| \varlimsup_{n \rightarrow \infty} \sqrt{\left|u_{n}\right|}<\infty .
$$


and hence the BI-summability is equivalent to ALsummability (sec. 3). From this remark and the implication $\mathrm{L} \rightarrow \mathrm{AL}$ it follows that series (52) can not be L-summable at an exterior point of $\pi$.

Let now $z_{0}$ be an interior point of $\pi$. Then the series $\sum_{v} u_{v} z_{0}^{v-1}$ is BI-summable and hence AL-summable. Thus the relation

$$
\lim _{\alpha \rightarrow 1-0}\left(\sum_{n} U_{n}\left(z_{0}\right) \alpha^{n}\right)=g\left(z_{0}\right)
$$

holds where $U_{n}\left(z_{0}\right)$ denotes the L-transform of the sequence $u_{v} z_{0}^{-1}$.

It remains to show that the series $\sum_{n} U_{n}\left(z_{0}\right)$ is convergent; then the relation

$$
\sum_{n} U_{n}\left(z_{0}\right)=g\left(z_{0}\right)
$$

will follow from (53) and the Abelian limit theorem.

We are going to prove the absolute convergence of the above series. For that we start out from the relations

$$
U_{n}\left(z_{0}\right)=\frac{1}{n !} \sum_{v=1}^{n-1} p_{n-1 v} u_{v+1} z_{0}^{v}
$$

Since $z_{0}$ is an interior point of the polygon $\pi$ the closed circle

$$
\left|z-\frac{z_{0}}{2}\right| \leqq \frac{\left|z_{0}\right|}{2}
$$

is contained in $D$, and therefore a number $\rho>1$ can be chosen so close to 1 that the circle

$$
c: \quad\left|z-\frac{z_{0}}{2}\right|=\rho \frac{\left|z_{0}\right|}{2}
$$

still is contained in $D$. [2, Chap. VIII.]

Hence the coefficients $u_{v}$ can be represented by

$$
u_{v}=\frac{1}{2 \pi i} \oint \frac{g(z)}{z^{v}} d z .
$$

From (54) and (55) we obtain

$$
U_{n}=\frac{1}{n !} \oint_{c} p_{n-1} v \frac{g(z)}{z^{v+1}} z_{0}^{v} d z=\frac{1}{n !} \oint_{c} \frac{g(z)}{z} p_{n-1}\left(\frac{z_{0}}{z}\right) d z
$$

and from there, if $M$ denotes the maximum of $\frac{g(z)}{z}$ on the circle $c$,

$$
\left|U_{n}\right| \leqq \frac{M}{n !}\left|p_{n-1}\left(\frac{z_{0}}{z}\right)\right|
$$

If we write for abbreviation

$$
\omega=\frac{z_{0}}{z},
$$

this relation becomes

$$
\begin{aligned}
& \left|U_{n}\right| \leqq \frac{M}{n !}|\omega||\omega+1| \ldots|\omega+n-2| . \\
& =M\left|\frac{\omega(\omega+1) \ldots(\omega+n)}{n ! n^{\omega}}\right| \cdot\left|\frac{n^{2}}{(\omega+n-1)(\omega+n)}\right| \cdot\left|n^{\omega-2}\right| .
\end{aligned}
$$

If $n$ tends to $\infty$ the first factor approaches $\Gamma(\omega)$ and therefore stays bounded. The same is true for the second term and therefore it is sufficient to show that the series

$$
\sum_{n}\left|n^{\omega-2}\right|
$$

is uniformly convergent on $c$. The circle $c$ is represented by the equation

$$
z=\frac{z_{0}}{2}\left(1+\rho e^{i \theta}\right) \quad(0 \leqq \theta \leqq 2 \pi) .
$$

From here it follows that

$$
\omega=\frac{z_{0}}{z}=\frac{2}{1+\rho e^{i \theta}}
$$

and for the real part

$$
R(\omega)=\frac{2(1+\rho \cos \theta)}{1+2 \rho \cos \theta+\rho^{2}} \leqq \frac{2}{1+\rho} .
$$

Therefore we have

$$
R(\omega-2) \leqq \frac{-2 \rho}{1+\rho}=-1-\delta, \quad(\delta>0)
$$

and hence the inequalities

$$
\left|n^{\omega-2}\right| \leqq n^{-(1+\delta)}
$$

hold, which imply the uniform convergence of series (57) on $c$. Now the absolute convergence of the series $\sum_{n} U_{n}$ follows from $(56)$.

\section{Connections With the Euler-Knopp Method}

The Euler-Knopp transform of a sequence $u_{v}$ (EK-transform) is defined to be the sequence

$$
U_{n}=\sum_{v}\left(\begin{array}{c}
n-1 \\
v-1
\end{array}\right) \kappa^{v}(1-\kappa)^{n-v} u_{v}
$$

where $\kappa$ is a complex parameter.

This transformation can be derived from a linear transformation of the $w$-plane which has the fixed 
point $\omega=1$ and maps $\omega=0$ into $\omega=\infty$. The most general transformation of this kind is given by

$$
z=\frac{1-a}{\omega}+a
$$

where $a$ is a complex number. Now let

$$
f(z)=\sum_{v} \frac{u_{v}}{z^{v}}
$$

be an analytic function which is regular for $|z|>1$. This implies that the function

$$
f^{*}(\omega)=f\left(\frac{1-a}{\omega}+a\right)
$$

is regular in the domain that is mapped onto $|z|>1$ under the transformation (59), that is the circle

$$
|1-a+a \omega|>|\omega| \text {. }
$$

Since this domain contains the point $\omega=0$ the function $f^{*}(\omega)$ can be expanded into a power series

$$
f^{*}(\omega)=\sum_{n} U_{n} \omega^{n}
$$

The coefficients $U_{n}$ are given by

$$
U_{n}=\frac{1}{2 \pi i} \oint \frac{f^{*}(\omega)}{\omega^{n+1}} d \omega
$$

If we substitute here for the function $f^{*}(\omega)$ the expression (61) and for $f(z)$ the expansion (60) we get

$$
U_{n}=\frac{1}{2 \pi i} \sum_{v} \oint\left(\frac{\omega}{1-a+a \omega}\right)^{v} \frac{d \omega}{\omega^{n+1}} u_{v} .
$$

This integral can be evaluated by the residue theorem. First we observe that

$$
\begin{aligned}
(1-a+a \omega)^{-v}=(1-a)^{-v}\left(1+\frac{a}{1-a}\right)^{-v} & \\
= & \kappa^{v}\{1+(\kappa-i) \omega\}^{-v}
\end{aligned}
$$

where

$$
\kappa=\frac{1}{1-a} .
$$

From there we obtain

$$
\begin{array}{r}
U_{n}=\frac{1}{2 \pi i}\left(\oint \sum_{v, n} \kappa^{v}\left(\frac{-v}{\mu}\right)(\kappa-1)^{\mu} \omega^{v+\mu-n-1} d \omega\right) u_{v} \\
=\sum_{v}\left(\begin{array}{c}
-v \\
n-v
\end{array}\right) \kappa^{v}(\kappa-1)^{n-v} u_{v}
\end{array}
$$

and making use of the identity

$$
\left(\begin{array}{c}
-v \\
n-v
\end{array}\right)(-1)^{n-v}=\left(\begin{array}{l}
n-1 \\
v-1
\end{array}\right),
$$

we finally get

$$
U_{n}=\sum_{v}\left(\begin{array}{l}
n-1 \\
v-1
\end{array}\right) \kappa^{v}(1-x)^{n-v} u_{v}
$$

This equation shows that the coefficients $U_{n}$ are the EK-transforms of the coefficients $u_{v}$.

From the relationship between the EK-transformation and the linear mapping (59) it can easily be derived that the EK-transformation cannot be regular unless $\kappa$ is real and $0<\kappa \leq 1$. Assume that transformation (58) is regular and let $\sum_{v} u_{v}$ be a convergent series such that the function

$$
f(z)=\sum_{v} \frac{v_{v}}{z_{v}}
$$

has a singularity at a given point $z_{1}\left(\left|z_{1}\right|=1, z_{1} \neq 1\right)$. Then the series $\sum_{n} U_{n}$ by hypothesis is also convergent and hence the function

$$
f^{*}(\omega)=f\left(\frac{1-\cdot a}{\omega}+a\right)
$$

is regular for $|\omega|<1$. Therefore the function $f(z)$ must be regular in the image domain of the circle $|\omega|<1$ under the mapping (59). This image is given by

$$
D_{a}:|z-a|>|1-a| .
$$

Therefore the point $z_{1}$ cannot be contained in $D_{a}$ and since $z_{1}$ was arbitrary on the unit circle this has to hold for the whole circle $|z|=1$. Hence the unit circle has to be contained in the circle

$$
|z-a| \leqq|1-a|
$$

and this is possible only if $a$ is real and $a \leqq 0$, which means that $\kappa$ is real and $0<\kappa \leqq 1$.

It is known that an EK-summable series is also AL-summable provided that $\kappa$ is real and positive [2, sec. 8.] We shall give here a proof of this fact using the integral representation of the L-transformation.

Let $\sum_{v} u_{v}$ be an EK-summable series and define the function $f(z)$ by

$$
f(z)=\sum_{v} \frac{u_{v}}{z_{v}}
$$


Then the function

$$
g(\omega)=f^{*}\left(\frac{1}{\omega}\right)=f((1-a) \omega+a)
$$

has the expansion

$$
g(\omega)=\sum_{n} U_{n} \omega^{n}
$$

The function $f(z)$ can be expressed by $g(\omega)$ as

$$
f(z)=g\left(\frac{z-a}{1-a}\right)
$$

From this equation it follows that the L-integral transform of $f(z)$ can be written as

$$
\begin{aligned}
& \phi(\alpha)=\frac{2}{2 \pi i} \oint \frac{(1-\alpha)^{1-z}-1}{z-1} f(z) d z \\
&=\frac{1}{2 \pi i} \oint \frac{(1-\alpha)^{1-z}-1}{z-1} g\left(\frac{z-a}{1-a}\right) d z .
\end{aligned}
$$

Introducing the new variable

$$
\zeta=\frac{z-a}{1-a}
$$

we obtain

$$
\phi(\alpha)=\frac{1}{2 \pi i} \oint \frac{(1-\alpha)^{(1-\zeta)(1-a)}-1}{\zeta-1} g(\zeta) d \zeta
$$

and hence $\phi(\alpha)$ can be considered as the transform of $g(\omega)$ with respect to the kernel

$$
\bar{K}(\zeta, \alpha)=\frac{(1-\alpha)^{(1-\zeta)(1-\alpha)}-1}{\zeta-1} .
$$

It is easily verified that this kernel fulfills the conditions of theorem 1 provided that $a$ is real and $0 \leqq a<1$. Therefore it follows that under this condition

$$
\lim _{\alpha \rightarrow 1-0} \phi(\alpha)=\sum_{n} U_{n}=\mathrm{EK}\left(\sum_{v} u_{v}\right)
$$

provided that the series $\sum_{n} U_{n}$ is convergent. But that means that the series $\sum_{v}^{n} u_{v}$ is AL-summable and the relation

$$
\mathrm{AL}\left(\sum_{v} u_{v}\right)=\mathrm{EK}\left(\sum_{v} u_{v}\right)
$$

holds.

In case $\kappa$ is not both real and positive it is shown by Agnew ([2], sec. 8) that EK-summability does not imply AL-summability.

\section{Inversion Formula of the Lototsky Integral Transformation}

The question arises whether the integral transformation

$$
\phi(\alpha)=\frac{1}{2 \pi i} \oint \frac{(1-\alpha)^{1-z}-1}{z-1} f(z) d z
$$

can be inverted. One cannot expect that to every given regular function $\phi(\alpha) \quad(|\alpha|<1)$ there exists a function $f(z)$ regular outside of a sufficiently large circle such that relation (65) holds. Provided such a function exists, it can be represented as an integral transform of $\phi(\alpha)$ at least in a half-plane.

Theorem 5. Let $f(z)$ be a function regular for $|z|>\mathrm{r}$ such that $f(\infty)=0$, and let $\phi(\alpha)$ be its transform with respect to $(65)$. Then in the half-plane $x>r$ the function $f(z)$ can be represented as

$$
f(z)=(z-1) \int_{0}^{1} \phi(\alpha)(1-\alpha)^{z-2} d \alpha .
$$

Proof: Since $f(\infty)=0$, relation (65) can be written as

$$
\phi(\alpha)=\frac{1}{2 \pi i} \oint \frac{(1-\alpha)^{1-z}}{z-1} f(z) d z
$$

Let $z_{0}=x_{0}+i y_{0}$ be a fixed point in the half-plane $x>r$. We choose for the path of integration in (65) a circle $|z|=R$, where

$$
r<R<x_{0} .
$$

If $(65)$ is multiplied by $(1-\alpha)^{z_{0}-2}$ and in tegrated with respect to $\alpha$ between 0 and $t(0<t<1)$, we get

$$
\int_{0}^{t} \phi(\alpha)(1-\alpha)^{z_{0}-2} d \alpha=\frac{1}{2 \pi i} \int_{0}^{t} \oint \frac{(1-\alpha)^{z_{0}-z-1}}{z-1} f(z) d z d \alpha .
$$

Since the integrand is regular, the two integrations may be interchanged and we obtain integrating with respect to $\alpha$

$$
\int_{0}^{t} \phi(\alpha)(1-\alpha)^{z_{0}-2} d \alpha=-\frac{1}{2 \pi i} \oint \frac{(1-t)^{z_{0}-z}-1}{(z-1)\left(z_{0}-z\right)} f(z) d z .
$$

If $t$ tends to 1 , the expression

$$
(1-t)^{z-z}
$$

tends to zero uniformly on $|z|=R$ because of the inequality

$$
\operatorname{Re}\left(z_{0}-z\right) \geqq x_{0}-R>0 .
$$

Hence we obtain

$$
\int_{0}^{1} \phi(\alpha)(1-\alpha)^{z_{0}-2} d \alpha=\frac{1}{2 \pi i} \oint \frac{f(z) d z}{(z-1)\left(z_{0}-z\right)} .
$$


The right-hand side of this equation can be evaluated by the Cauchy integral formula since the function $f(z)$ is regular for $|z|>r$. Observing that $f(\infty)=0$, we get

$$
\frac{1}{2 \pi i} \oint \frac{f(z) d z}{(z-1)\left(z_{0}-z\right)}=\frac{f\left(z_{0}\right)}{z_{0}-1} .
$$

From (67) and (68) it follows that

$$
\frac{f\left(z_{0}\right)}{z_{0}-1}=\int_{0}^{1} \phi(\alpha)(1-\alpha)^{z_{0}-2} d \alpha
$$

and we obtain the formula

$$
f(z)=(z-1) \int_{0}^{1} \phi(\alpha)(1-\alpha)^{z-2} d \alpha \quad(x>r)
$$

which is the inversion of (65).

Formula (69) can be used to obtain the inverse matrix of the L-transformation (13). For the particular functions

$$
\phi(\alpha)=\alpha^{k} \quad(k=0,1 \ldots)
$$

we get from (69)

$$
\begin{aligned}
f(z)=(z-1) \int_{0}^{1} \alpha^{k}(1-\alpha)^{z-2} d \alpha & \\
& =\frac{k !}{z(z+1) \ldots(z+k-1)}=\frac{k !}{p_{k}(z)} .
\end{aligned}
$$

This function is in fact regular outside of the circle $|z|=k-1$ and thus for the special functions (70) the integral equation (65) does have a solution that is regular outside of a certian circle.

To obtain the inverse of the L-transformation

$$
U_{n}=\frac{1}{n !} \sum_{v} p_{n-1 v-1} u_{v}
$$

we denote by $q_{n j}$ the inverse of the matrix $p_{n j}$. Then it follows from (71) that

$$
u_{n}=\sum_{i} j ! q_{n-1 j-1} U_{j}
$$

The functions

$$
\phi(\alpha)=\sum_{n} U_{n} \alpha^{n}
$$

and

$$
f(z)=\sum_{v} \frac{u_{v}}{z^{v}}
$$

correspond to each other by the transformations $(65)$ and (68).

In particular, the function (70) possesses the coefficients

$$
U_{n}=\delta_{n k},
$$

and hence we obtain from (72)

$$
u_{n}=\sum_{j} j ! q_{n-1 j-1} \delta_{j k}=k ! q_{n-1 k-1} .
$$

Thus the function $f(z)$ has the expansion

$$
f(z)=k ! \sum_{n} \frac{q_{n-1 k-1}}{z^{n}} .
$$

On the other hand this function is given by

$$
f(z)=\frac{k !}{p_{k}(z)}
$$

Comparing the right-hand sides of (73) and (74) we conclude that

$$
\sum_{n} \frac{q_{n-1 k-1}}{z^{n}}=\frac{1}{p_{k}(z)}
$$

and from there

$$
q_{n-1 k-1}=\frac{1}{2 \pi i} \oint \frac{z^{n-1}}{p_{k}(z)} d z
$$

or if $\mathrm{n}-1$ is replaced by $n$ and $k-1$ by $k$,

$$
q_{n k}=\frac{1}{2 \pi i} \oint \frac{z^{n}}{p_{k+1}(z)} d z .
$$

This formula corresponds to the representation (31) of the matrix $p_{n v}$. If the integral in $(75)$ is evaluated by the residue theorem we obtain the formula

$$
\begin{aligned}
q_{n k}=\sum_{v=1}^{k}(-1)^{n-v} \frac{v^{n-1}}{(v-1) !(k-v) !} \\
=\frac{1}{(k-1) !} \sum_{v=1}^{k}(-1)^{n-v}\left(\begin{array}{c}
k-1 \\
v-1
\end{array}\right) v^{n-1} .
\end{aligned}
$$

\section{References}

[1] R. P. Agnew, The Lototsky method for evaluation of series, Mich. Math. J. 4 (1957).

[2] G. H. Hardy, Divergent series (Oxford, England, 1949).

[3] A. V. Lototsky, On a linear transformation of sequences and series, Ivanov. Gos. Ped. Inst. Uc. Fiz.-Mat. Nauki 4, 61 (1953) (in Russian).

Washington, D.C.

(Paper 63B1-1) 